\title{
Caregivers of family members with dementia were involved in a dynamic decision making process to maintain tolerable situations
}

Wackerbarth S.Modeling a dynamic decision process: supporting the decisions of caregivers of family members with dementia. Qual Health Res 1999 May;9:294-314.

QUESTION: How do family caregivers of people with dementia make decisions?

\section{Design}

Multiple case study approach.

\section{Setting}

Wisconsin, USA.

\section{Participants}

28 caregivers (mean age 62 y, $60 \%$ women, $54 \%$ adult children, $40 \%$ spouses) were interviewed. Duration of providing care ranged from $<6$ months to $>10$ years. $71 \%$ of care receivers (mean age 80 y, $75 \%$ women) lived in a care facility, $14 \%$ lived at home alone, and $14 \%$ lived with the caregiver.

\section{Methods}

20 caregivers were initially interviewed in their homes for 45 minutes to 2 hours. They were asked about when they first recognised the signs of dementia and about some of the challenging decisions they had made and how they had made them. Interview transcripts were analysed to identify characteristics of the caregiver's decision making process; these were plotted on a timeline. Common themes emerged during cross-informant comparisons, and an overall model was developed. 2 cycles of focus group review, theoretical modification, and 8 more interviews were used to validate the model. The 28 caregivers who were interviewed also provided feedback on the model.

\section{Main findings}

Decision making can be modelled around the "intrapersonal struggle in which caregivers reacted to and balanced their caregiving situation.” The model (fig) incorporates the element of time and 3 main components: a tolerance line, status points, and decision events. Time refers to the dynamic nature of the decision making process and starts at the point that caregiving first begins. Previous situations (status points) and decision events influence how caregivers react to future situations.

The tolerance line is the upper limit of what a caregiver perceives to be a tolerable situation. In general,

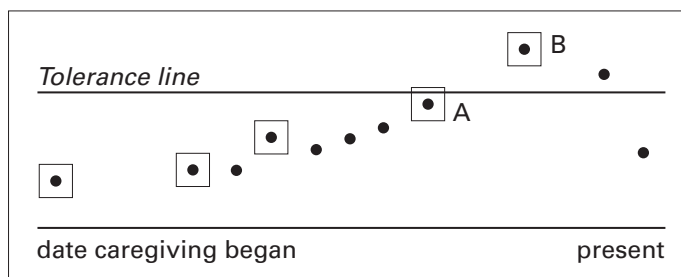

The dynamic model of the caregiving decision process. $(\bullet$ status points; $\square$ decision events.) Reprinted with permission from

Wackerbath S. Modeling a dynamic decision process: supporting the decisions of caregivers of family members with dementia. Qual Health Res 1999;9:294-314. caregivers wanted family members to remain at home or have as much freedom as possible. Small adjustments to make this happen were acceptable as long as caregiver control was maintained.

Status points are plotted across time and fall above or below the tolerance line. They represent a caregiver's assessment of how tolerable a situation is during an episode. As the status points get closer to the tolerance line, the caregiving situation gets more difficult, to the point of being intolerable if the points are above the tolerance line.

A status point becomes a decision event if a situation leads a caregiver to consider taking action. Decisions are made (1) to react to a crisis - that is, make the situation more tolerable while avoiding or delaying a more drastic change; and (2) to prepare for the future, usually when caregivers recognise that their caregiving ability has diminished.

\section{Conclusion}

The decision making process of caregivers of family members with dementia was depicted in a complex and dynamic model that focused on maintaining tolerability in the caregiving situation.

\section{COMMENTARY}

This study by Wackerbarth uses the caregiving burden perspective to address decision making in caregiving for individuals with dementia. It provides useful information about how 28 caregivers made decisions and what formal support was and was not beneficial. Decision making is seen as another burden, a perspective that ignores research on the positive aspects of and the meaning gained through caregiving. ${ }^{2}$.

The validity of the emergent model was enhanced by triangulation of 3 sources of data, and the detailed account of this process will be useful to other researchers.

The proposed model of decision making is useful to healthcare providers. For example, the model depicts the tensions between the tolerance level of the caregiver and the need to care for the care recipient at home. This can be instructive for providers of care to attend to tolerance levels, status points, and decision events. The authors caution, however, against prejudging what a given caregiver should do. A good example is "pressuring" the caregiver to put the recipient of care into a facility.

Wackerbarth also identified 5 decision styles used by the caregivers in this study: planning ahead, taking it one day at a time, having difficulty implementing decisions, receiving decision support from service providers, and feeling that caregiving decisions are made by others or the disease itself. This information will help providers to support the caregiver better by matching information and suggestions to these decision styles. It also explains why some caregivers get support from groups and others do not and why there is anger and frustration when certain solutions or actions advocated by individual providers or groups, such as the Alzheimer's Society, are not acceptable. Anna Marie Hughes, RN, EdD Assistant Professor, School of Nursing University of British Columbia Vancouver, British Columbia, Canada

1 Farran C, Keane-Hagerty E, Salloway S, et al. Finding meaning: an alternative paradigm for Alzheimer's disease family caregivers. The Gerontologist 1991;31:483-9.

2 Perry J. A study of women caregiving to husbands who have Alzheimer's disease: family know-how as a process of interpretive caring. Unpublished doctoral dissertation, University of Washington, 1995. 